\title{
Multiphoton Antiresonance and Quantum Activation in Driven Systems
}

\author{
M. I. Dykman \\ Department of Physics and Astronomy, Michigan State University, East Lansing, MI 48824, USA
}

\begin{abstract}
We show that nonlinear response of a quantum oscillator displays antiresonant dips and resonant peaks with varying frequency of the driving field. The effect is a consequence of special symmetry and is related to resonant multiphoton mixing of several pairs of oscillator states at a time. We also discuss escape from a metastable state of forced vibrations. Two important examples show that the probability of escape via diffusion over quasienergy is larger than via dynamical tunneling provided the relaxation rate exceeds both of them. Diffusion dominates even for zero temperature, so that escape occurs via quantum rather than thermal activation. The effects can be studied using Josephson junctions and Josephson-junction based systems.
\end{abstract}

\section{INTRODUCTION}

Much progress has been made recently in experimental studies of periodically modulated vibrational systems. Examples include optically bistable systems, electrons in Penning traps, Josephson junctions, and various nano- and micro-mechanical resonators [1]-[8]. All these systems display bistability of forced vibrations. Because of thermal or externally applied noise, there occurs switching between coexisting stable vibrational states. The measured switching probabilities of noise-induced transitions are in a good agreement with the theoretical predictions.

In the present paper we are interested in the dynamics of a quantum oscillator. The generality of the oscillator as a model system and the current interest in quantum computing and coherent phenomena lead to two major questions: (i) does a resonantly driven oscillator display coherent quantum effects that would qualitatively differ from those in two-level systems, and (ii) in the presence of relaxation, what is the probability of switching between coexisting stable states due to quantum fluctuations? These two questions are addressed in the present paper, which is based on the results [9]-[11].

A weakly nonlinear oscillator is a multi-level quantum system with nearly equidistant energy levels $E_{n}$. Therefore a periodic force of frequency $\omega_{F}$ can be nearly resonant for many transitions at a time, i.e., $\hbar \omega_{F}$ can be close to the interlevel distance $E_{n+1}-E_{n}$ for many $n$. This leads to strong nonlinearity of the response even to comparatively weak resonant fields. A well-known quantum effect of the oscillator nonlinearity is the onset of Rabi oscillations due to resonant multiphoton transitions. Multiphoton Rabi oscillations occur when the spacing between remote energy levels $n$ and $m$ coincides with the energy of $n-m$ photons, $E_{n}-E_{m}=(n-m) \hbar \omega_{F}$ [12]. The multiphoton transition amplitude is resonantly enhanced, because an $m \rightarrow n$ transition occurs via a sequence of virtual fieldinduced transitions $k \rightarrow k+1$ (with $m \leq k \leq n-1$ ), all of which are almost resonant. An 
associated classical effect, in the presence of dissipation, is hysteresis of the amplitude of forced vibrations as function of the field amplitude $A$ and $\omega_{F}$.

In this paper (see also [10]) we show that multiphoton transitions in the oscillator are accompanied by a new effect, antiresonance of the response. When the frequency of the driving field adiabatically passes through a resonant value, the vibration amplitude displays a sharp minimum or maximum, depending on the initially occupied state. We argue that the antiresonance and the multiphoton Rabi oscillations can be observed in Josephson junctions.

If the frequency $\omega_{F}$ is close to twice the oscillator frequency, then $\hbar \omega_{F}$ is close to $E_{n+2}-E_{n}$ for many $n$ at a time. This leads to parametric resonance in the oscillator, in which it oscillates at frequency $\omega_{F} / 2$ in response to the driving. Such oscillations are intrinsically bistable, because their phase can take on two values that differ by $\pi$.

We will be most interested in the semiclassical behavior of the oscillator, which, on the one hand, stretches all the way to the classical region, and on the other hand, works well for oscillators even deep in the quantum domain. In the semiclassical picture, resonant multiphoton transitions correspond to tunneling between Floquet states of the oscillator with equal quasienergies. [The quasienergy $\varepsilon$ gives the change of the wave function $\psi(t)$ when time is incremented by the modulation period $\tau_{F}, \psi(t+$ $\left.\left.\tau_{F}\right)=\exp \left(-i \varepsilon \tau_{F} / \hbar\right) \psi(t)\right]$. The occurrence of equal-quasienergy states is related to the bistability of forced vibrations of a classical oscillator.

Tunneling of a driven oscillator is a carefully studied[13] example of dynamical tunneling [14]. The WKB analysis gives an important insight into the origin of the antiresonance, which goes beyond the perturbation theory in the driving field.

Dynamical tunneling also leads to transitions between coexisting metastable states of forced vibrations, which emerge in the presence of dissipation due to coupling to a thermal reservoir. In terms of quantum mechanics, dissipation is due to interlevel oscillator transitions with energy being transferred to (or from, for nonzero temperature) the reservoir. It turns out that dissipation may also lead to transitions between metastable states of forced vibrations, even for zero temperature [9].

For $T=0$ there occur only interlevel transitions where the oscillator energy goes to the reservoir (but the energy loss is compensated by the driving field, in the stationary regime). However, the quasi-energy may increase or decrease as a result of a coupling-toreservoir induced transition, although with different probabilities. Therefore along with drift over quasienergy towards a metastable state, which results from more probable transition, there emerges diffusion away from this state as a sequence of less probable transitions. The diffusion may lead to activated-like escape. Activation in this case has purely quantum nature, and therefore we call it quantum activation.

\section{THE MODELS}

The Hamiltonian of a nonlinear oscillator with mass $M=1$ has the form

$$
H(t)=\frac{1}{2} p^{2}+\frac{1}{2} \omega_{0}^{2} q^{2}+\frac{1}{4} \gamma q^{4}+H_{F}(t) .
$$


We will consider two types of periodic modulation, $H_{F}=H_{F}^{(r, p)}$, which correspond to resonant and parametric driving,

$$
\begin{aligned}
& H_{F}^{(r)}(t)=-q A \cos \left(\omega_{F} t\right), \quad \delta \omega^{(r)} \equiv \omega_{F}-\omega_{0} \ll \omega_{0}, \\
& H_{F}^{(p)}=\frac{1}{2} q^{2} F \cos \left(\omega_{F} t\right), \\
& \delta \omega^{(p)} \equiv \frac{1}{2} \omega_{F}-\omega_{0} \ll \omega_{0}
\end{aligned}
$$

(in what follows we set $\gamma>0$ ).

It is convenient to analyze the dynamics in the rotating wave approximation by switching from the fast oscillating operators $q, p$ to slowly varying operators $Q, P$ using transformations

$$
q=\alpha^{(r)}\left(Q \cos \omega^{(r)} t+P \sin \omega^{(r)} t\right), \quad p=-\alpha^{(r)} \omega^{(r)}\left(Q \sin \omega^{(r)} t-P \cos \omega^{(r)} t\right)
$$

for resonant driving and

$$
q=\alpha^{(p)}\left(P \cos \omega^{(p)} t-Q \sin \omega^{(p)} t\right), \quad p=-\alpha^{(p)} \omega^{(p)}\left(P \sin \omega^{(p)} t+Q \cos \omega^{(p)} t\right)
$$

for parametric driving, with

$$
\omega^{(r)}=\omega_{F}, \quad \alpha^{(r)}=\left(8 \omega_{F} \delta \omega^{(r)} / 3 \gamma\right)^{1 / 2}
$$

and

$$
\omega^{(p)}=\omega_{F} / 2, \quad \alpha^{(p)}=(2 F / 3 \gamma)^{1 / 2} .
$$

The variables $Q, P$ are the appropriately scaled coordinate and momentum. The commutation relation for them has a simple form

$$
[P, Q]=-i \lambda, \quad \lambda=\hbar\left(\omega^{(r, p)}\right)^{-1}\left(\alpha^{(r, p)}\right)^{-2}
$$

The parameter $\lambda$ plays the role of the effective Planck constant. We note that it is proportional to the oscillator nonlinearity $\gamma$ scaled by the comparatively small detuning of the field frequency, in the case of nearly resonant driving, or the comparatively small field amplitude, in the case of parametric driving.

The dynamics of $Q, P$ in the two cases is described by effective Hamiltonians

$$
\begin{gathered}
H^{(r)}=\omega_{F} \delta \omega^{(r)}\left(\alpha^{(r)}\right)^{2} g^{(r)}, \\
H^{(p)}=(F / 4)\left(\alpha^{(p)}\right)^{2} g^{(p)} .
\end{gathered}
$$

Their eigenvalues are equal to the quasienergies $\varepsilon_{n}$ of the oscillator. The functions $g^{(r, p)}$ have the forms

$$
\begin{array}{r}
g^{(r)}(P, Q)=\frac{1}{4}\left(Q^{2}+P^{2}-1\right)^{2}-\beta^{1 / 2} Q, \quad \beta=\frac{3 \gamma A^{2}}{32 \omega_{F}^{3}\left(\delta \omega^{(r)}\right)^{3}} \\
g^{(p)}=\frac{1}{4}\left(Q^{2}+P^{2}\right)^{2}+\frac{1}{2}(1-\mu) P^{2}-\frac{1}{2}(1+\mu) Q^{2}, \quad \mu=2 \omega_{F} \delta \omega^{(p)} / F .
\end{array}
$$


They are shown in Fig. 1 Each of them depends on one parameter. In the region of bistability of period one vibrations, $0<\beta<4 / 27$, the function $g^{(r)}$ has a shape of a tilted Mexican hat, with a maximum at the top of the central dome and a minimum at the lowest point of the rim. For a parametrically excited oscillator in the region $-1<\mu<1$ the function $g^{(p)}$ has two symmetrical minima. These extrema of $g^{(r, p)}$ correspond to metastable states of the driven oscillator in the presence of weak dissipation. The saddle points of $g^{(r, p)}$ correspond to unstable states of forced vibrations.
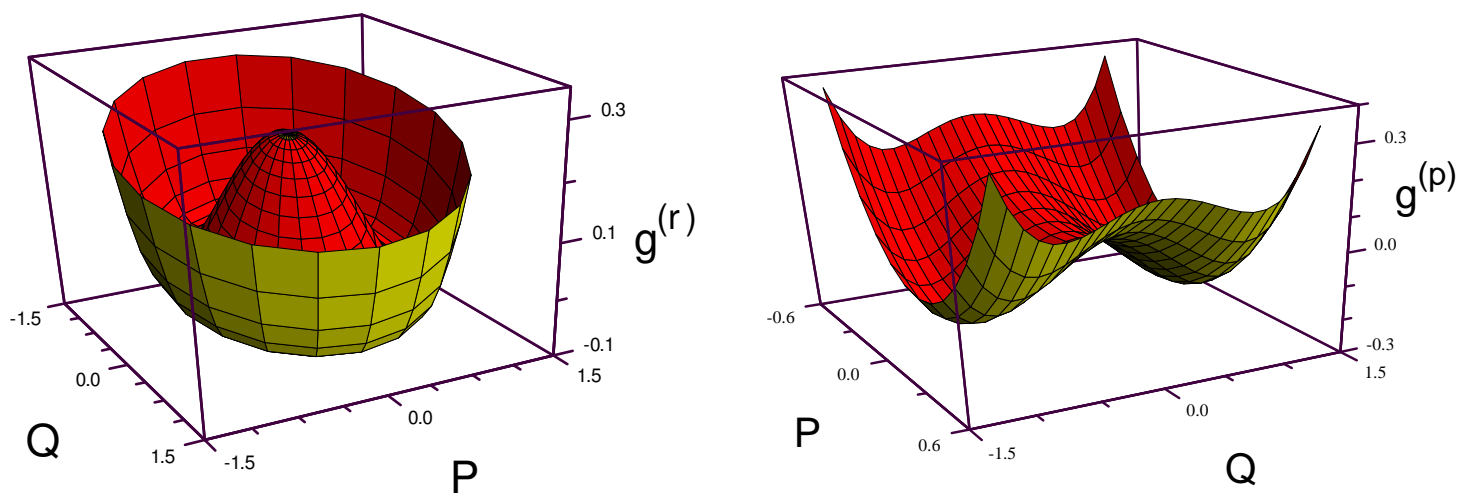

FIGURE 1. The scaled classical quasienergy of the oscillator for resonant and parametric driving (left and right panels, respectively) as function of the slowly varying coordinate and momentum $Q, P$. The plot of $g^{(r)}$ refers to the reduced field intensity $\beta=3 \gamma A^{2} / 32 \omega_{F}^{3}\left(\delta \omega^{(r)}\right)^{3}=1 / 27$. The plot of $g^{(p)}$ refers to $\mu=2 \omega_{F} \delta \omega^{(p)} / F=-0.1$.

\section{MULTIPHOTON ANTIRESONANCE}

We will start with the studies of the coherent response of the oscillator to a nearly resonant field. When the field amplitude $A \rightarrow 0$, the eigenstates $|n\rangle$ of the Hamiltonian $H^{(r)}$ coincide with the Fock states of the oscillator, and the quasienergies are

$$
\varepsilon_{n}=-\delta \omega^{(r)} n+\frac{1}{2} V n(n+1), \quad V=\frac{3 \hbar \gamma}{4 \omega_{0}^{2}} .
$$

We keep only the lowest-order term in $V$, which corresponds to the weak nonlinearity approximation. In this approximation the energy of an $N$ th oscillator state for $A=0$ is $E_{N}=\hbar \omega_{0} N+V N(N+1) / 2$. The $N$-photon resonance $N \hbar \omega_{F}=E_{N}-E_{0}$ occurs, in terms of $\delta \omega^{(r)}$, for

$$
\delta \omega^{(r)}=\delta \omega_{N}^{(r)}=V(N+1) / 2 .
$$

For the corresponding field frequency $\omega_{F}$ the quasienergies $\varepsilon_{0}$ and $\varepsilon_{N}$ are equal.

The field leads to mixing of the wave functions of resonating states and to level anticrossing. This anticrossing is clearly seen in the upper right panel of Fig. 2 The minimal splitting of the levels $\varepsilon_{0}$ and $\varepsilon_{N}$ is given by the multiphoton Rabi frequency $\Omega_{R}$. For weak field it can be obtained by perturbation theory [12]. To the lowest order in the 
field amplitude $A$ in the limit of large $N$

$$
\Omega_{R}=V\left(A / A_{N}\right)^{N} N^{5 / 4}(2 \pi)^{-3 / 4}, \quad A_{N}=\left(2 \hbar \omega_{0}\right)^{1 / 2}|V| N^{3 / 2} \exp (-3 / 2) / 2 .
$$

The Rabi frequency depends on $N$ exponentially, $\Omega_{R} \propto A^{N}$. This dependence works well numerically in the whole region $A<A_{N}$ [10].
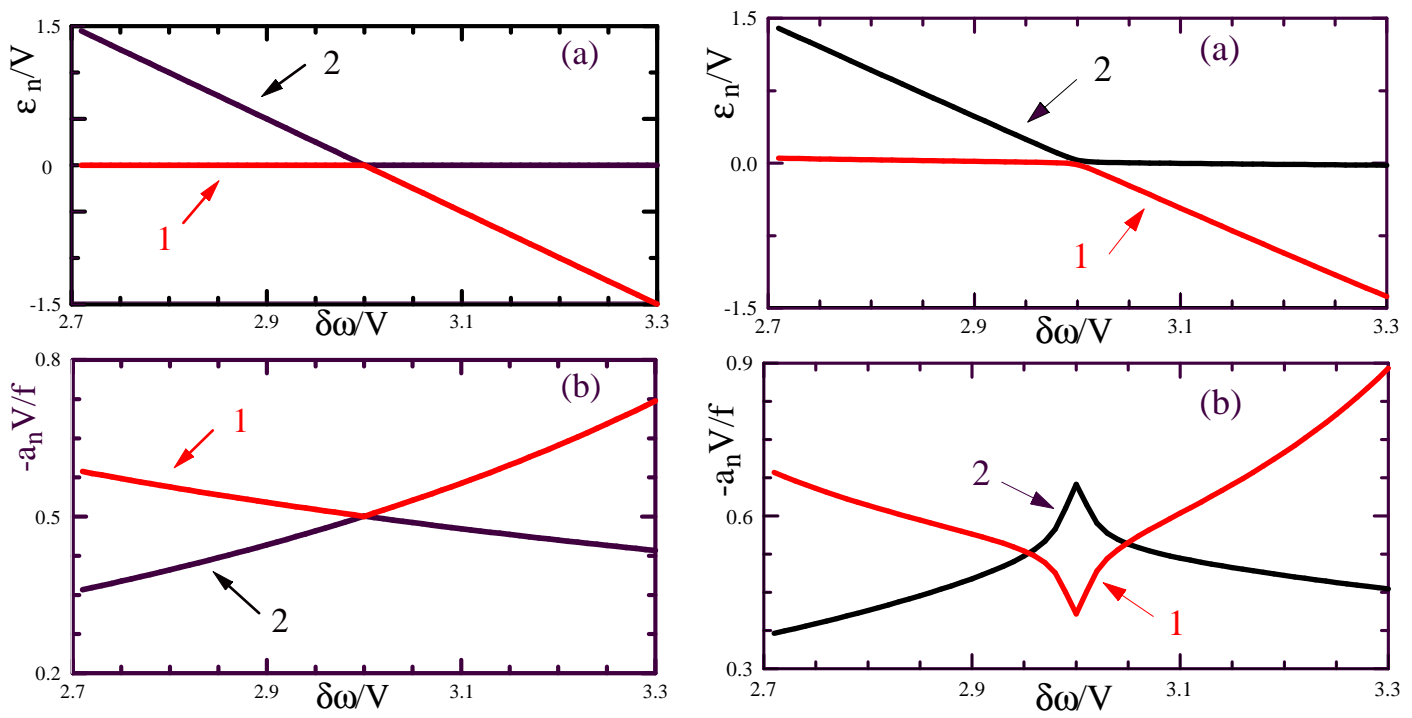

FIGURE 2. Quasienergy levels $\varepsilon_{n}$ and reduced susceptibilities in the resonating states of a driven nonlinear oscillator. The plots refer to a 5-photon resonance, $N=5$. The labels 1 and 2 correspond to the ground $(n=0)$ and 5 th $(n=5)$ oscillator states in the absence of driving, for $\delta \omega \equiv \delta \omega^{(r)}<\delta \omega_{5}^{(r)}$. Left panel: the limit of zero driving amplitude, $A \rightarrow 0$. The quasienergy levels cross for $\delta \omega_{N}^{(r)} / V=3$. The susceptibilities in the resonating states also cross at that same frequency. Right panel: level repulsion and antiresonance of susceptibilities for comparatively weak modulation. The data refer to $A / A_{N}=1 / 2$. It is seen from the figure that the susceptibility antiresonance is much more pronounced than the level repulsion. The scaling factor for the amplitude $a_{n}$ of oscillator vibrations in the states $n=0,5$ is $f=$ $\left(8 \hbar \omega_{0}\right)^{-1 / 2} A$.

Coherent response of the oscillator to the driving field is characterized by the expectation value of the coordinate $q$. If the oscillator is in an eigenstate $|n\rangle$ of the Hamiltonian $H^{(r)}$, this value has the form

$$
q_{n}=\left(\hbar / 2 \omega_{0}\right)^{1 / 2} a_{n} e^{-i \omega_{F} t}+\text { c.c. }, \quad q_{n}=\langle n|q| n\rangle .
$$

To first order in the field, the reduced amplitude of forced vibrations in an $n$th state $a_{n}$ is

$$
a_{n}=-f \delta \omega^{(r)} /\left(\left(\delta \omega^{(r)}-V n\right)\left[\delta \omega^{(r)}-V(n+1)\right]\right), \quad f=\left(8 \hbar \omega_{0}\right)^{-1 / 2} A .
$$

Remarkably, for $\delta \omega^{(r)}=\delta \omega_{N}^{(r)}$ the vibration amplitudes in the resonating states coincide with each other, $a_{N-n}=a_{n}$ for $0 \leq n<N / 2$, see the left lower panel in Fig. [2]

Field-induced multiphoton mixing leads not only to splitting of the quasienergy levels, but also to repulsion of the vibration amplitudes. It can be calculated by diagonalizing 
the Hamiltonian $H^{(r)}$ and is shown in the right lower panel of Fig. 2 as a function of frequency detuning $\delta \omega \equiv \delta \omega^{(r)}$. One of the involved resonating states is the ground state of the oscillator $n=0$ in the limit $A \rightarrow 0$. The quantities plotted in Figs. 2(b) are susceptibilities, they are proportional to the ratio of the vibration amplitude to the modulation amplitude $a_{n} / A$.

The antiresonant splitting of the expectation values of the vibration amplitudes is by far the most interesting feature of Fig. 2. It occurs at the adiabatic passage of $\delta \omega^{(r)}$ through resonance, where the system switches between the ground and excited states. In particular, the amplitude displays an antiresonant dip if the oscillator is mostly in the ground state for $\left(\delta \omega^{(r)}-\delta \omega_{N}^{(r)}\right) / V<1$ or in the state $N$ for $\left(\delta \omega^{(r)}-\delta \omega_{N}^{(r)}\right) / V>1$. The magnitude and sharpness of the dip are determined by $\Omega_{R} / V$ and depend very strongly on the field and $N$. With decreasing $\Omega_{R} / V$ the dip (and peak) start looking like cusps located at resonant frequency. We note that, in contrast to the case of energy levels, there is no reason for repulsion (anticrossing) of susceptibilities. In fact, as seen from Fig. 2 the susceptibilities do cross, although away from the resonant frequency. The effect of antiresonance is due purely to specific quantum interference [10].

The dip in the oscillator response has no analogue in two-level systems. There, for nearly resonant driving, the coherent response in the two adiabatic states differs only in sign. It displays a peak when the radiation frequency adiabatically passes through the transition frequency.

\section{The WKB picture of the antiresonance}

In the WKB approximation, Rabi oscillations correspond to tunneling between the states with nearly equal quasienergies. Such semiclassical states can be found from the Hamiltonian $H^{(r)}$ (4) by using the Bohr-Sommerfeld quantization condition applied to the mechanical action $\oint P d Q$ for trajectories $g(Q, P)=$ const, with $\hbar$ replaced by $\lambda$, Eq. (3). It is seen from the left panel of Fig. 1 that, in a certain range of $g$, there are two types of trajectories with the same $g$, those on the internal dome and those on the external part of the Mexican hat $g(Q, P)$. If, as a result of the Bohr-Sommerfeld quantization, the quantized values of $g$ on the two parts of the surface $g(Q, P)$ coincide with each other, then there may occur resonant tunneling between the corresponding quantum states. The resulting tunneling splitting [13] is the Rabi frequency.

Interestingly, one can show that the average value of the coordinate

$$
Q(g)=\tau^{-1}(g) \int_{0}^{\tau(g)} Q(t) d t
$$

$[\tau(g)$ is the period of oscillations for a given $g]$ is the same for the internal and external trajectories with the same $g$. This corresponds to the susceptibilities of the resonating quasienergy states being the same, in the neglect of tunneling-induced mixing of the states. We emphasize that the fact that the susceptibilities are equal is not a result of the perturbation theory in the field amplitude, as in the case of Eq. (10), they are equal in all orders of the perturbation theory in $A$ as long as tunneling is disregarded. Tunnelinginduced state mixing leads to the antiresonance of the response [10]. 


\section{ESCAPE OF A DRIVEN SYSTEM: TUNNELING OR QUANTUM ACTIVATION?}

We will now briefly outline the new effects and unanswered questions that emerge when dissipation is taken into account [9, 11]. We will assume that, even though dissipation is weak, the dissipation rate exceeds the tunneling rate. The problem of fairly general interest that will be addressed is switching between metastable states of forced vibrations of a quantum oscillator. We will consider the most interesting situation where there are many quasienergy states between the extrema of the quasienergy surface. In the case of escape of a particle from a potential well it corresponds to a well with many energy levels.

In systems in thermal equilibrium, the rate of tunneling decay of a metastable state for low temperatures is given by the probability of a tunneling transition from the ground state in a metastable potential well. In the case of a resonantly driven oscillator this corresponds to dynamical tunneling from the top of the dome of the quasienergy surface $g^{(r)}$ to the state on the external orbit with the same quasienergy, see the left panel of Fig. 1. The tunneling is shown schematically in the central panel of Fig. 3. For a parametrically driven oscillator the corresponding tunneling occurs between the minima of the surface $g^{(p)}$, as shown in the right panel of Fig. 3.

For higher temperatures, again in the case of equilibrium systems, one has to take into account tunneling from excited intrawell states. Escape may occur also via thermal activation over the potential barrier. One of these escape mechanisms dominates, depending on temperature [15]. In the case of a driven oscillator tunneling from excited states corresponds to tunneling with quasienergies that differ from those at the extrema of $g(Q, P)$. In addition, there is a probability of activation over the quasienergy barrier. However, since the distribution over quasienergy is not of the Boltzmann form, it is not clear which of the escape mechanisms dominates at a given temperature of the bath.

For weak dissipation the distribution can be described by the balance equation for the occupations $\rho_{n}$ of quasienergy (Floquet) states $|n\rangle$,

$$
\dot{\rho}_{n}=-\sum_{m} W_{n m} \rho_{n}+\sum_{m} W_{m n} \rho_{m}
$$

The transitions probabilities $W_{n m}$ can be calculated as matrix elements of the operator that describes relaxation of the oscillator. The wave functions $|n\rangle$ can be found from the Bohr-Sommerfeld approximation disregarding tunneling. A standard WKB calculation allows one to express $W_{n m}$ in terms of the Fourier components of the coordinate and momentum $Q_{m-n}\left(g_{n}\right), P_{m-n}\left(g_{n}\right)$ for a given quasienergy $g_{n}$ [the functions $Q(t), P(t)$ are periodic functions of time for a given $g$, with period $\tau(g)]$. In the simple case of linear friction, which corresponds to relaxation transitions between nearest energy (not quasienergy) levels of the oscillator shown in the left panel of Fig. 3, $W_{n m}$ are simply quadratic in $Q_{m-n}\left(g_{n}\right), P_{m-n}\left(g_{n}\right)$. They exponentially decay with $|n-m|$.

The probabilities $W_{n m}$ are organized so that it is more likely for a system to make a transition toward the value of $g$ in the metastable state rather than away from it. This is why the state is metastable. However, in contrast to systems in equilibrium, the probabilities $W_{n m}$ do not satisfy the condition $W_{n m}=W_{m n} \exp \left[\left(g_{n}-g_{m}\right) / k T\right]$. Even for $T \rightarrow 0$ there is a nonzero probability to make a transition in the direction opposite to the 
metastable state. This is a consequence of the fact that the Floquet states $|n\rangle$ are linear combinations of the Fock states of the oscillator. Therefore, even where all transitions between the Fock states go in one direction in energy, as in the left panel of Fig. 3 . transitions between the Floquet states go in different directions in quasienergy, although with different probabilities.
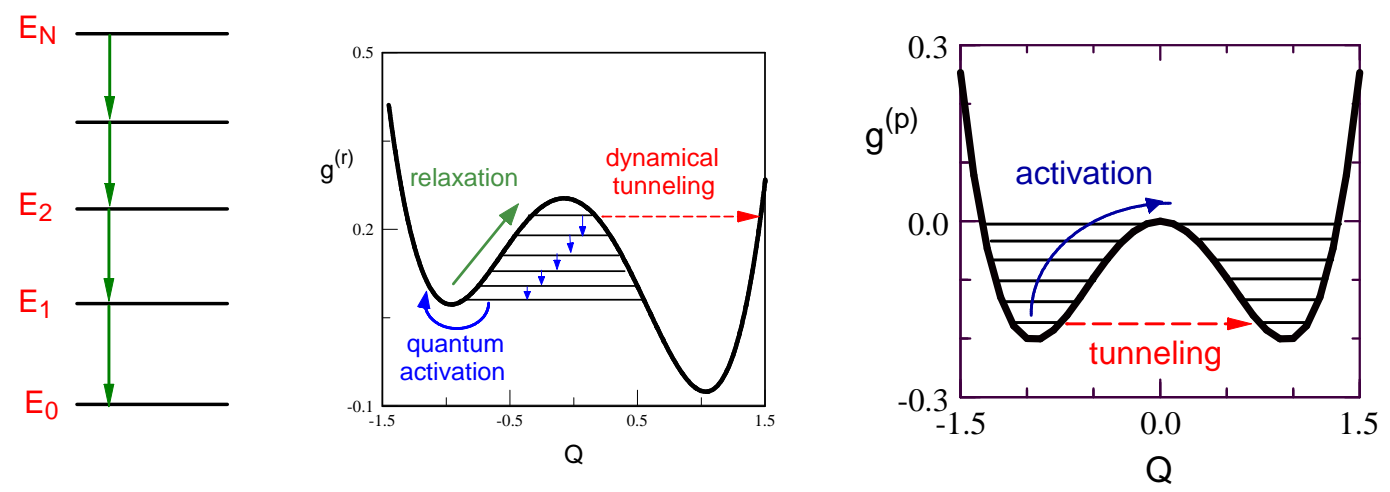

FIGURE 3. Left panel: transitions between the energy levels of the oscillator $E_{n}$ due to coupling to a thermal reservoir. For $T=0$ there occur only transitions to lower levels with emission of excitations of the reservoir. In the simplest case relaxation is due to transitions between neighboring oscillator levels. Central panel: the cross-section of the surface of the scaled quasienergy $g^{(r)}$ shown in Fig. 11 by the plane $P=0$. Quasienergy levels for orbits that lie on the dome in Fig. 1 are shown schematically. Relaxation to the metastable state at the top of the dome corresponds to transitions to higher quasienergy, whereas less probable transitions to lower quasienergy lead to an activation-type escape from the metastable state. Right panel: the cross-section of the quasienergy surface of the parametrically driven oscillator $g^{(p)}$, Fig. 1] by the plane $P=0$, with schematically shown quasienergy levels. Relaxation drives the oscillator down to the lowest quasienergy levels at the bottom of the wells of $g^{(p)}(Q, P)$, but there is a possibility of activation even for $T=0$.

Transitions in the "wrong" direction lead to diffusion over quasienergy away from the metastable state. Their immediate consequence is a finite width of the stationary distribution over quasienergy even for $T \rightarrow 0$. Another closely related consequence is a nonzero probability to reach quasienergy of the saddle of the functions $g^{(r, p)}$ starting from a metastable state. The saddle of $g^{(r, p)}$ is similar to the top of a potential barrier for a particle in a metastable potential. The logarithm of the probability of reaching the saddle gives the activation energy of escape as a result of diffusion over quasienergy. We call this process quantum activation, since it occurs even for $T=0$.

For resonantly and parametrically driven oscillators the transition rates $W_{n m}$ can be calculated explicitly using the fact that the classical trajectories in $(Q, P)$-variables can be expressed in terms of the Jacobi elliptic functions [9, 11]. We have compared the activation energies of escape with the tunneling exponents. When the activation energy is smaller than the tunneling exponent in the absolute value, escape occurs via quasienergy diffusion. We found that, both for a resonantly and a parametrically excited oscillator, escape from metastable states occurs via activation, not tunneling. This holds true for all parameter values where a nonlinear oscillator has coexisting stable states. We found an unusual behavior of the distribution for an underdamped parametrically driven oscillator for $T=0$. For some quasienergy the distribution displays a sharp decrease, and at the same time the tunneling rate goes to zero. 
The physical origin of the fact that escape occurs via activation, not dynamical tunneling, remains not fully understood, the existing arguments are formal [9]. Apparently, there must be a crossover from activation to tunneling when the system goes to equilibrium, but the models that we have discussed are strongly nonequilibrium, the very presence of coexisting metastable states is due to periodic driving.

In conclusion, we have shown that a simple system, a driven nonlinear oscillator, displays unusual quantum coherent phenomena and unusual switching behavior. The considered effects have no analogue in two-level systems and are qualitatively different from what has been known about switching in thermal equilibrium systems. They are not only of fundamental interest, but are also important for many applications, in particular in sensing and quantum measurements.

\section{ACKNOWLEDGMENTS}

This research was supported in part by the Institute for Quantum Sciences at Michigan State University and by the NSF through grant ITR-0085922.

\section{REFERENCES}

1. P. D. Drummond and D. F. Walls, J. Phys. A 13, 725 (1980).

2. $\quad$ G. Gabrielse, H. Dehmelt, and W. Kells, Phys. Rev. Lett. 54, 537 (1985).

3. A.I. Larkin and Yu.N. Ovchinnikov, J. Low Temp. Phys. 63, 317 (1986); B.I. Ivlev and V.I. Mel'nikov, Phys. Lett. A 116, 427 (1986); S. Linkwitz and H. Grabert, Phys. Rev. B 44 11888, 11901 (1991); M.H. Devoret, D. Esteve, J.M. Martinis, A. Cleland, and J. Clarke, Phys. Rev. B 36, 58-73 (1987).

4. A. Wallraff, T. Duty, A. Lukashenko, and A. V. Ustinov, Phys. Rev. Lett. 90, 037003 (2003); M. V. Fistul, A. Wallraff, and A. V. Ustinov, Phys. Rev. B, 68, 060504(R) (2003).

5. I. Siddiqi, R. Vijay, F. Pierre, C. M. Wilson, M. Metcalfe, C. Rigetti, L. Frunzio, and M. H. Devoret, Phys. Rev. Lett. 93, 207002 (2004).

6. J. S. Aldridge and A. N. Cleland, Phys. Rev. Lett. 94, 156403 (2005); cond-mat/0406528 (2004).

7. R. L. Badzey, G. Zolfagharkhani, A. Gaidarzhy, and P. Mohanty, Appl. Phys. Lett. 86, 023106 (2005).

8. C. Stambaugh and H. B. Chan, cond-mat/0504791(2005).

9. M.I. Dykman and V.N. Smelyanskiy, Zh. Eksp. Teor. Fiz. 94, 61 (1988) [Sov. Phys. JETP 67, 1769 (1988)].

10. M.I. Dykman and M.V. Fistul, Phys. Rev. B 71, 140508(R) (2005).

11. M.I. Dykman and M. Marthaler, unpublished.

12. D.M. Larsen and N. Bloembergen, Opt. Communications 17, 254 (1976).

13. A.P. Dmitriev and M.I. D'yakonov, Zh. Eks. Teor. Fiz. 90, 1430 (1986) [Sov. Phys. JETP 63, 838 (1986)].

14. M. J. Davis and E. J. Heller, J. Chem Phys. 75, 246 (1981).

15. A. I. Larkin and Y. N. Ovchinnikov, J. Stat. Phys. 41, 425 (1985) 